\title{
Single-particle motion under the influence of the perpendicular ponderomotive force
}

\author{
By M. C. FESTEAU-BARRIOZ†, M. L.SAWLEY \\ AND J. VÁCLAVIK
}

Centre de Recherches en Physique des Plasmas, Association Euratom - Confédération Suisse, Ecole Polytechnique Fédérale de Lausanne, 21, Av. des Bains,

CH-1007 Lausanne, Switzerland

(Received 7 July 1986 and in revised form 11 September 1986)

The motion of a single particle under the influence of the ponderomotive force directed perpendicular to the external magnetostatic field is analysed. By solving the exact equation of motion for a specific applied electromagnetic field, the resultant ponderomotive drift is compared with the prediction of a single-particle theory using the oscillation-centre approximation. The regime of validity of this theory is discussed. It is shown that, for certain values of the amplitude and frequency of the electromagnetic field, the particle motion is unstable and therefore the concept of a single-particle ponderomotive force is meaningless.

\section{Introduction}

It is well known that a high-frequency electromagnetic field may exert on a charged particle a time averaged (ponderomotive) force. The resultant particle motion is generally analysed by means of the oscillation-centre approximation (Motz \& Watson 1967), in which the position of the particle is split into slowly and rapidly varying parts, $x=x_{s}+x_{f}$. Here $x_{s}$ describes the time averaged motion of the oscillation centre, and $x_{f}$ the fast motion about the oscillation centre. Assuming that $\left|\mathbf{x}_{f}\right| \ll\left|\mathbf{x}_{s}\right|$, the equation of motion can be separated according to slow and fast time scales. Solving the equation of motion on the slow time scale yields the time averaged motion of the particle under the influence of the ponderomotive force. In the presence of an external magnetostatic field $\mathbf{B}_{0}$, the ponderomotive force parallel to $\mathbf{B}_{0}$ gives rise to acceleration in the direction along $\mathbf{B}_{\mathbf{0}}$, while the perpendicular ponderomotive force leads to a mutually perpendicular particle drift.

The concept of the ponderomotive force has received much attention in the field of plasma physics owing to its application to the confinement and acceleration of plasmas (Motz \& Watson 1967). Recent studies have investigated, for example, RF plugging (Watari et al. 1978; Fader et al. 1981) and low-frequency mode stabilization (Ferron et al. 1983; Yasaka \& Itatani 1984) in mirror devices. Since the collective nature of a plasma under the influence of an RF field may play an important role in determining the plasma dynamics, a single-particle treatment may not, however, always be appropriate. Indeed,

† Present address : Centre de Calcul, Ecole Polytechnique Fédérale de Lausanne, Ecublens, 1015 Lausanne, Switzerland. 
it has been shown (Klíma 1968; Statham \& ter Haar 1983) that the ponderomotive force obtained from a fluid description of the plasma does not agree with that obtained using the oscillation-centre approximation described above. A detailed discussion of the origin of this fundamental difference between the fluid and single-particle ponderomotive forces may be found elsewhere (Sawley \& Václavík 1986; Václavík, Sawley \& Anderegg 1986).

It may be asked whether the fluid result for the slow drift caused by the perpendicular ponderomotive force could be obtained from single-particle theory if the oscillation-centre approximation is not used. That this is not possible can be shown most convincingly by comparing the fluid result with the solution of the exact equation of motion for a single particle. In the present paper, a specific example of single-particle motion under the influence of a perpendicular ponderomotive force is considered. By numerical integration of the equation of motion, we compare the exact solution with that obtained from the application of the oscillation-centre approximation. We are thus able to show that single-particle theory is not capable of yielding the fluid result.

The use of the oscillation-centre approximation to yield the slow motion of the particle tacitly assumes that the amplitude of the fast motion is small. However, as this paper will show, the fast motion can in fact become unstable for certain values of frequency and amplitude of the RF field. Under these conditions, the concept of a single-particle ponderomotive force is, of course, meaningless.

\section{Mathematical formulation}

\subsection{Equation of motion}

In this paper we shall consider the motion of a single particle of charge $q$ and mass $m$ under the combined influence of an electromagnetic field and a uniform magnetostatic field $\left(\mathbf{B}_{0}=\hat{e}_{z} B_{0}\right)$. For the sake of simplicity we shall consider the specific example for which the total electric and magnetic fields may be written as

$$
\mathbf{E}(x, t)=-\hat{e}_{y} B_{0} \tilde{b} x, \quad \mathbf{B}(t)=\hat{e}_{z} B_{0}(1+\tilde{b}),
$$

where $\tilde{b}=b \cos \omega t$ and $b$ is constant. We shall ignore the self-consistent contribution of the total fields due to the motion of the charged particle.

The equation of motion for the particle in the above fields is

$$
\begin{gathered}
\ddot{x}=\Omega(1+\tilde{b}) \dot{y}, \\
\ddot{y}=-\Omega \tilde{b} x-\Omega(1+\tilde{b}) \dot{x}, \\
\ddot{z}=0,
\end{gathered}
$$

where the cyclotron frequency $\Omega=q B_{0} / m$. The particle motion perpendicular to $\mathbf{B}_{0}$ is thus described mathematically in terms of two coupled differential equations. It should be noted that these equations are nonlinear in the RF field amplitude but, for the present choice of fields, linear in the particle position.

In the following, we shall normalize quantities by adopting $\Omega^{-1}$ and $p \Omega^{-1}$ as time and distance units. The most appropriate choice for the normalizing velocity $p$ will become apparent in $\S 2.3$. We shall use the normalized quantities

$$
X=\Omega x / p, \quad Y=\Omega y / p, \quad T=\Omega t \quad \text { and } \quad \nu=\omega / \Omega .
$$




\subsection{Oscillation-centre approximation}

Splitting the particle motion perpendicular to $B_{0}$ into slowly and rapidly varying parts,

$$
\mathbf{X}_{\perp}=\hat{e}_{x}\left(X_{s}+X_{f}\right)+\hat{e}_{y}\left(Y_{s}+Y_{f}\right)
$$

(2) and (3) may be separated according to slow and fast time scales, as is usual in the oscillation-centre approximation (Motz \& Watson 1967). The fast time scale equation is solved to $O(b)$ to yield

$$
\left.\begin{array}{c}
X_{f}=-\frac{2 b X_{s}}{\left(1-\nu^{2}\right)} \cos \frac{(1-\nu) T}{2} \cos \frac{(1+\nu) T}{2} \\
Y_{f}=\frac{2 \nu b X_{s}}{\left(1-\nu^{2}\right)} \cos \frac{(1-\nu) T}{2} \sin \frac{(1+\nu) T}{2} .
\end{array}\right\}
$$

In general, a linear combination of $\sin T$ and $\cos T$ must be added to solutions (7) in order to satisfy the initial conditions for the particle fast motion. However, this yields no contribution to the slow (time averaged) motion, which is given to $O\left(b^{2}\right)$ by

$$
\frac{d X_{s}}{d T}=0, \quad \frac{d Y_{s}}{d T}=-\frac{\nu^{2} b^{2}}{2\left(1-\nu^{2}\right)} X_{s}
$$

From (8) it can be seen that the ponderomotive force perpendicular to $\mathbf{B}_{\mathbf{0}}$ gives rise to a time averaged drift in the $y$ direction. It may be readily shown that this drift can be written, as is usual, in terms of the single-particle ponderomotive potential:

$$
\frac{d Y_{s}}{d T}=\frac{d \phi_{p}}{d X_{s}} \quad \text { where } \backslash \phi_{p}=-\frac{\left|\mathrm{E}\left(X_{s}, T\right) / B_{0}\right|^{2}}{4\left(1-\nu^{2}\right)} .
$$

\subsection{Exact solution}

Equations (2) and (3) may be numerically integrated to yield the exact solution for the particle motion perpendicular to $\mathbf{B}_{0}$. However, using the Hamiltonian formulation, since $y$ is an ignorable co-ordinate the $y$ component of the canonical momentum, $P_{y}=m p$, is a constant of motion. It is straightforward to show that (2) and (3) may therefore be written, in normalized form, as

$$
\begin{gathered}
\left(d^{2} X / d T^{2}\right)=(1+\tilde{b})-(1+\tilde{b})^{2} X, \\
(d Y / d T)=1-(1+\tilde{b}) X .
\end{gathered}
$$

Equation (10), which describes the $x$ component of the particle motion, may be recognized as an inhomogeneous Hill equation.

Equations (10) and (11) must be supplemented by an appropriate set of initial conditions. For definiteness, we consider

$$
X(0)=1, \quad \frac{d X}{d T}(0)=0 \quad \text { and } \quad Y(0)=0 .
$$

This initial value problem may be solved by standard Runge-Kutta numerical integration techniques, to yield the exact nonlinear motion of the particle under the influence of the electromagnetic field. 

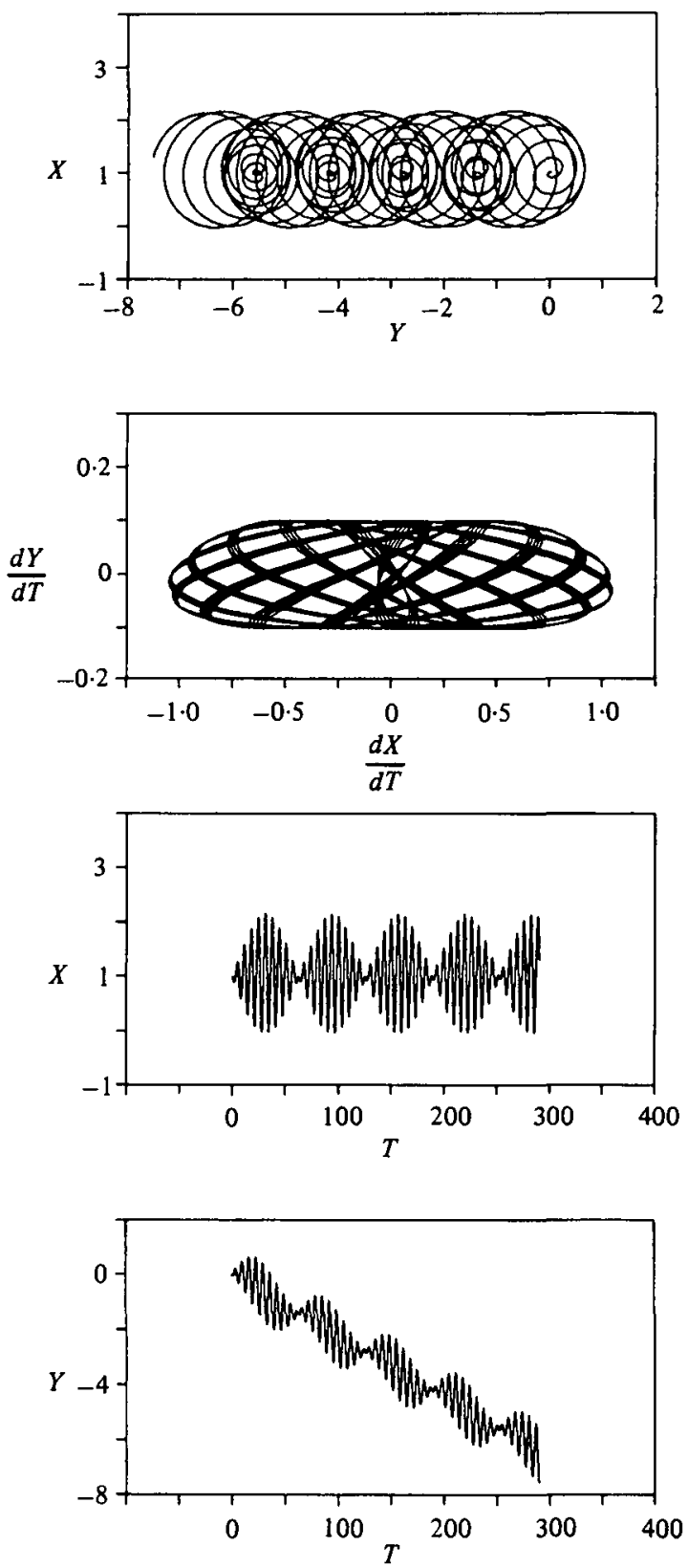

Figure 1. Particle motion for $\nu=0.9$ and $b=0 \cdot 1$.

\section{Results}

\subsection{Particle motion}

The initial value problem defined by (10)-(12) has been numerically solved for a number of different values of $v$ and $b$. The solution for a particular example $(\nu=0.9, b=0.1)$ is shown in figure 1 . Under the influence of the fields, the particle exhibits a fast orbital motion that is amplitude modulated at an intermediate frequency $v_{m}$. The slow time scale (ponderomotive) drift of the particle in the (negative) $y$ direction is also apparent. 

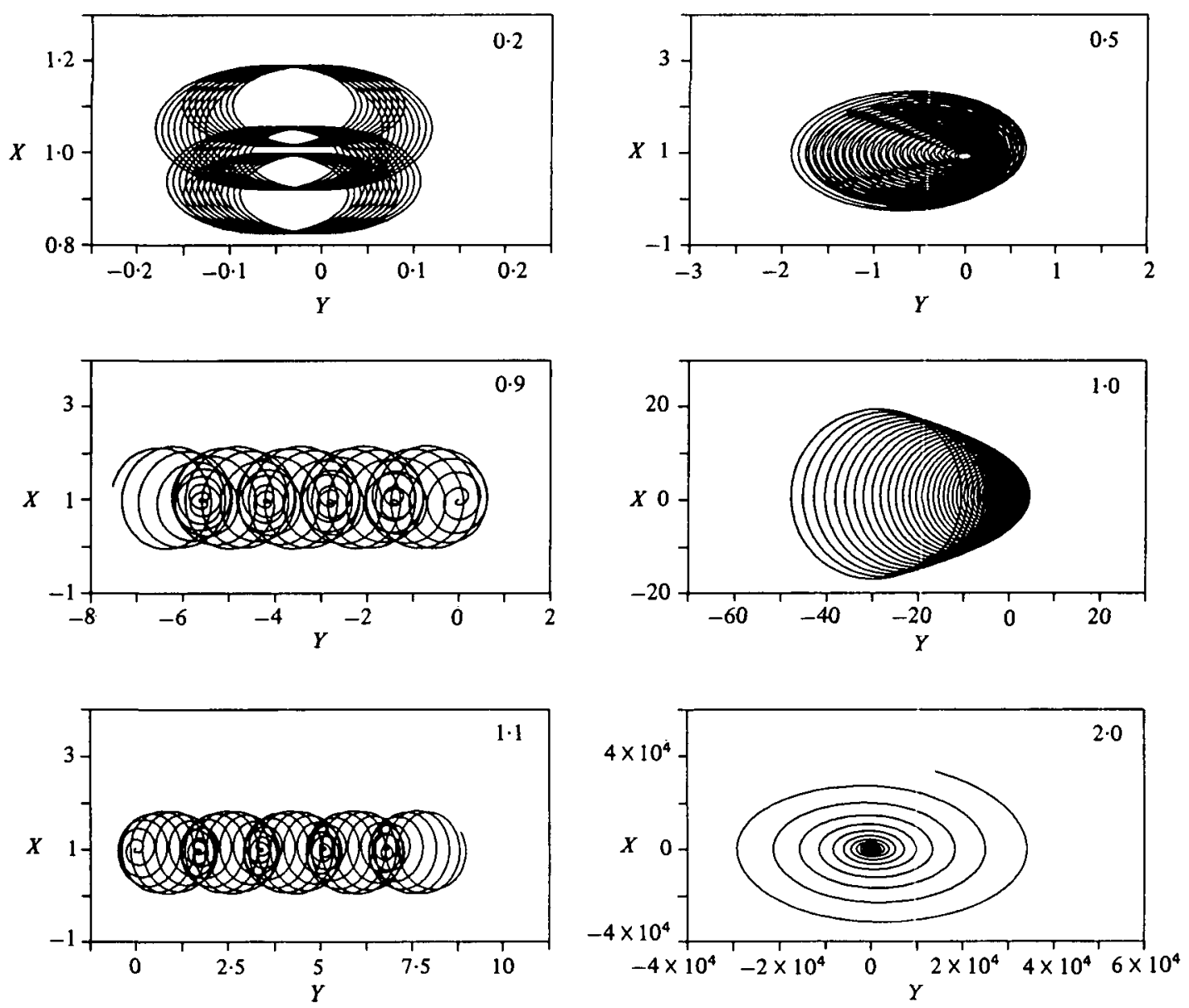

FIgURe 2. Particle motion for $b=0.1$ and for the six indicated values of $\nu$. The three examples on the left represent stable orbits, while the three on the right are unstable. The total time is the same $(T=290)$ for all examples.

The effect of changing the frequency $\nu$ of the electromagnetic field is demonstrated in figure 2. For the six cases shown, the amplitude of the field is $b=0.1$. It may be observed that the particle motion is strongly modified by a change of frequency. For certain of the values shown $(\nu=0 \cdot 5,1 \cdot 0,2 \cdot 0)$, the motion is unstable; the amplitude of the fast component increasing without bound. Comparing the cases $\nu=0.9$ and $1 \cdot 1$, it may be seen that the direction of the ponderomotive drift changes sign as the frequency crosses the cyclotron frequency $(\nu=1)$. For both of these cases, the amplitudes of the fast motion and ponderomotive drift are larger than for the case $\nu=0.2$ for which the frequency is further from the cyclotron frequency.

Figure 3 illustrates the effect on the particle motion of a change in the field amplitude while keeping the frequency constant $(\nu=0.9)$. As the field amplitude is increased, the amplitude of both the fast motion and the ponderomotive drift increases. For $b=0 \cdot 6$, the motion of the particle is no longer stable. Also evident from figure 3 is that as $b$ is increased from 0.1 to 0.5 , the frequency $v_{m}$ at which the fast motion is modulated decreases. 

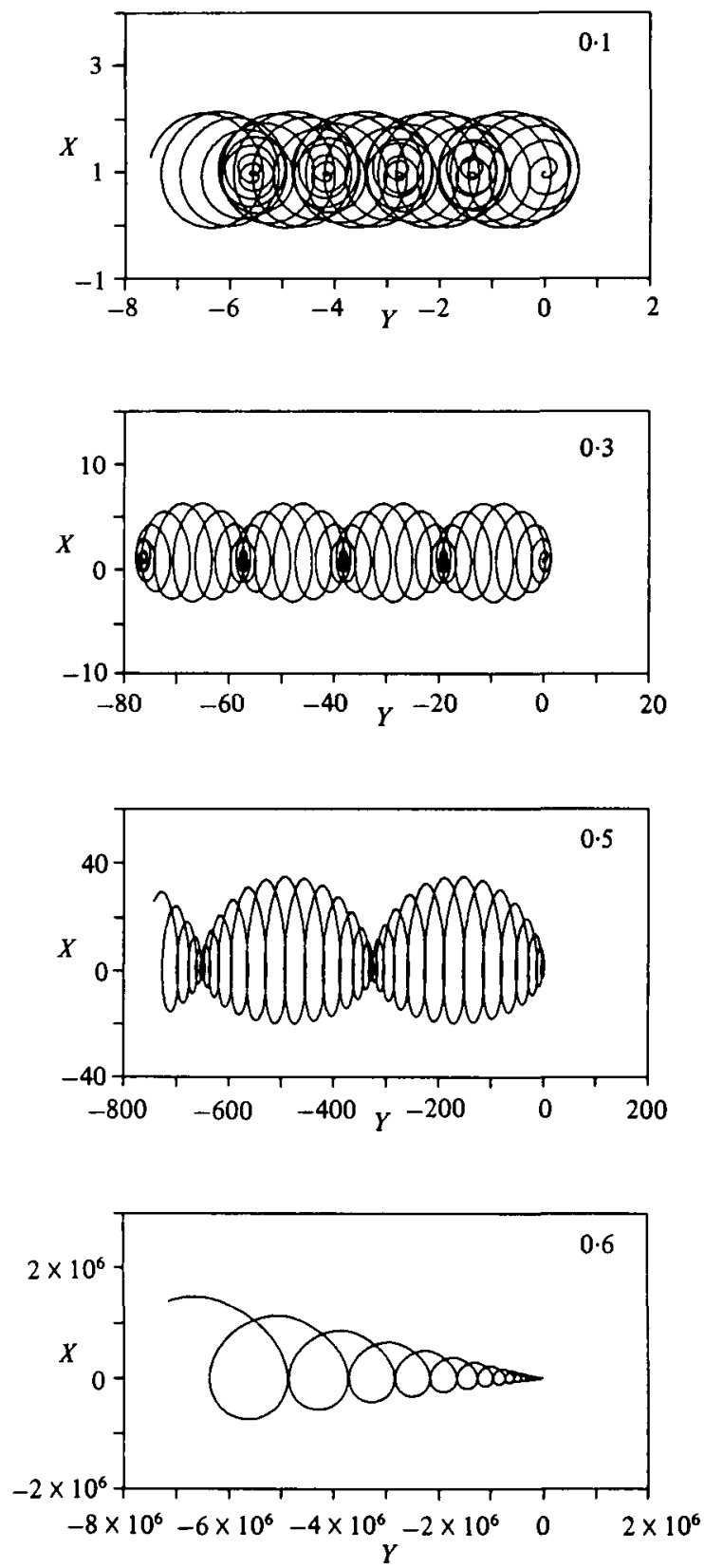

Fiaure 3. Particle motion for $v=0.9$ and for the four indicated values of $b$. The total time is the same $(T=290)$ for all examples.

\subsection{Comparison with the oscillation-centre approximation}

Except for particular values of field frequency $\nu$, the numerically calculated particle motion agrees qualitatively with that predicted using the oscillationcentre approximation provided that the field amplitude $b$ is sufficiently small. A more quantitative comparison can be obtained from figures 4-6.

In figure 4 is plotted, for three values of $\nu$, the modulation frequency $v_{m}$, 


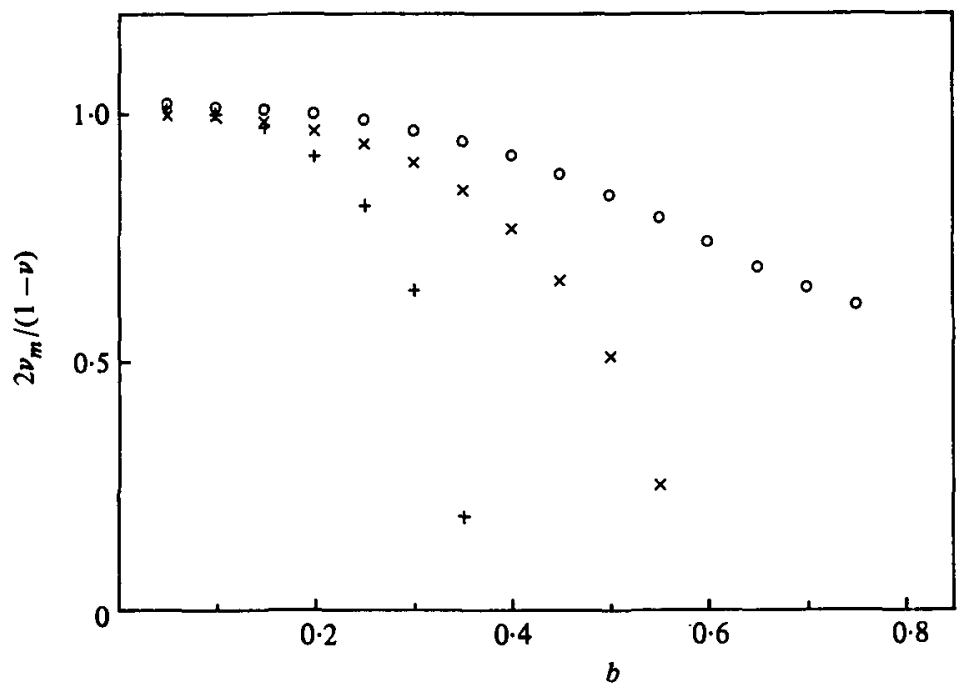

Figure 4. Normalized modulation frequency as a function of $b$, for $\bigcirc, \nu=0.85$;

$$
\times, \nu=0.9 ;+, \nu=0.95 \text {. }
$$

normalized to the values $\frac{1}{2}(1-v)$ determined using the oscillation-centre approximation, as a function of $b$. For $b \ll 1, \nu_{m} \simeq \frac{1}{2}(1-\nu)$ as is expected. However, as $b$ is increased, a sharp drop in $\nu_{m}$ is observed, being most pronounced for $v$ close to 1 . The decrease of $v_{m}$ to zero results from approaching the instability boundary: further increase in the field amplitude increases the growth rate of the unstable motion.

The effect of the field amplitude on the magnitude of the ponderomotive drift velocity is shown in figure 5 . In this figure are plotted values of the ponderomotive drift velocity $d Y_{s} / d T$, for three values of $\nu$, obtained from both numerical integration and the oscillation-centre approximation, (8). Again it may be seen that for $b \ll 1$ the two calculations yield similar results. However, as $b$ increases, so does the discrepancy between the results. When the particle motion is unstable, the concept of a ponderomotive drift becomes meaningless.

Figure 6 shows a plot, for $b=0 \cdot 2$, of the frequency dependence of the value of the ponderomotive drift velocity obtained from numerical integration, normalized to that calculated using the oscillation-centre approximation. The points plotted in this figure are for values of $\nu$ and $b$ for which the fast motion of the particle is stable. It is found that unstable orbits occur in the vicinity of

$$
\nu=(2 / m) \text { for integer } m \text {. }
$$

The width of these regions of instability increases with the value of $b$, and is largest for small values of $m$.

From figure 6 it may also be seen that the values of the ponderomotive drift velocity obtained by the two methods diverge if

$$
\nu=(1 / n) \text { for integer } n .
$$

Clearly, for these values of frequency, the application of the oscillation-centre approximation is not valid. Note that the ponderomotive drift velocity arising 


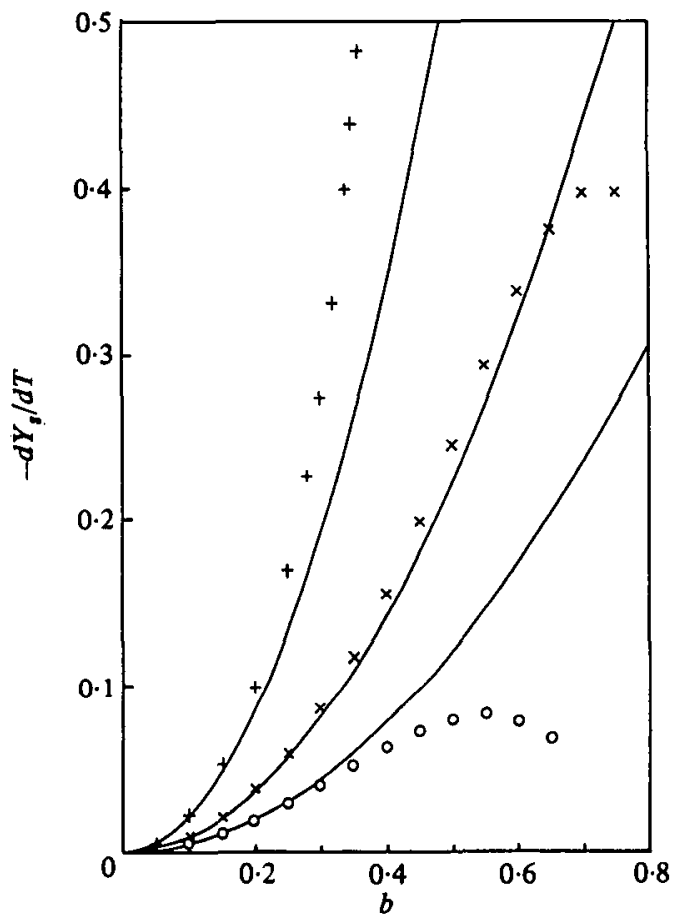

Figure 5. Ponderomotive drift velocity as a function of $b$, for $O, \nu=0.7 ; \times, \nu=0.8 ;+$, $\nu=0 \cdot 9$. corresponding curves obtained using the oscillation-centre approximation, (8).

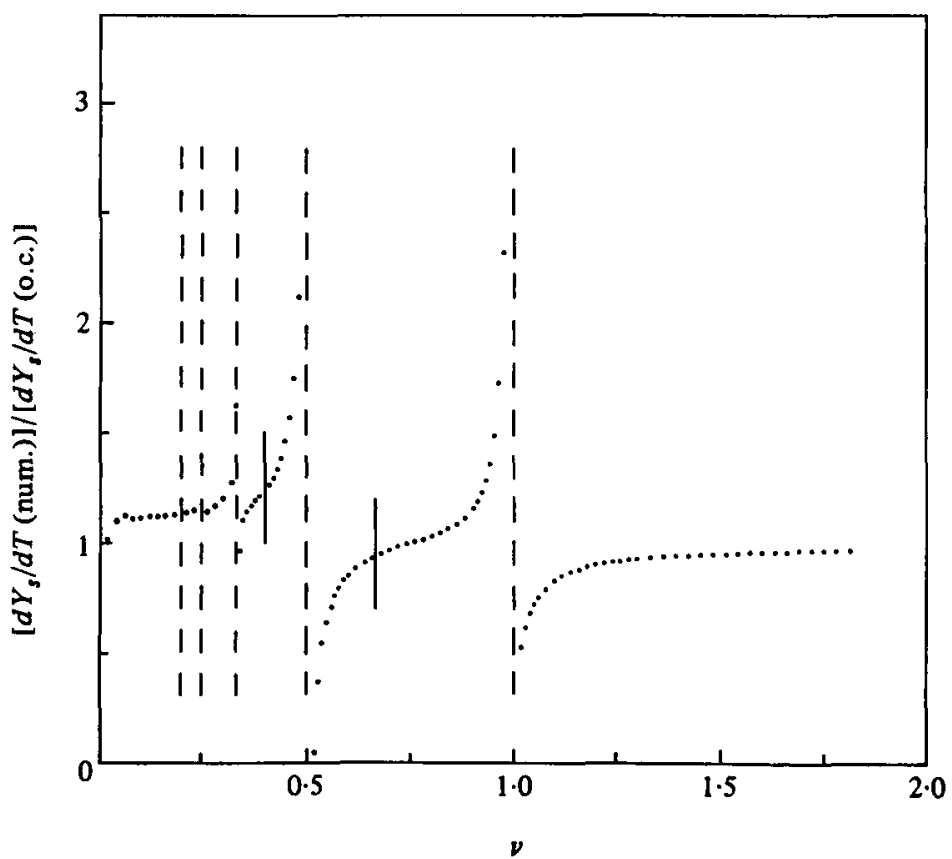

Frgure 6. Normalized ponderomotive drift velocity as a function of $\nu$ for $b=0 \cdot 2$. ---, values of $\nu$ satisfying (14); — , values of $\nu$ satisfying (13) for odd $m$. 
from stable orbits near the frequencies given by (13), with $m$ odd, are well described using the oscillation-centre approximation.

The origin of the regions of instability can be appreciated by noticing that for $b$ sufficiently small, (10) becomes an inhomogeneous form of the Mathieu equation (McLachlan 1964):

$$
\frac{d^{2} X}{d Z^{2}}+(a-2 q \cos 2 Z) X=0
$$

where

$$
Z=\frac{\nu T}{2}, \quad a=\frac{4}{v^{2}} \quad \text { and } \quad q=-\frac{4 b}{v^{2}}
$$

The well-known stability diagram for the Mathieu equation reveals unstable regions which, for small $q$, are in the vicinity of $a=m^{2}$ for integer $m$. This condition can be seen to be equivalent to (13).

In the oscillation-centre approximation, the equation of motion is solved to second order in $b$ to obtain the ponderomotive drift. However, if $b$ is nonnegligible, higher-order terms may yield a significant contribution. These higher-order contributions to the ponderomotive drift are particularly important if the frequency of the applied electromagnetic field is a sub-harmonic of the cyclotron frequency. The nonlinearity of the equation of motion leads, in this case, to oscillatory motion at the cyclotron frequency, resulting in a resonant ponderomotive contribution. Thus if (14) is approximately satisfied, the oscillation-centre approximation will yield an inaccurate calculation of the full nonlinear ponderomotive force.

\section{Conclusions}

By analysing in detail a particular example, the motion of a single particle under the influence of an electromagnetic field has been investigated. It has been shown that the particle motion is strongly modified by a change in the electromagnetic field amplitude (if sufficiently large) or the wave frequency (if in the vicinity of the cyclotron frequency).

Comparison has been made of the ponderomotive drift obtained using the oscillation-centre approximation and from numerical integration of the equation of motion. Within the regime of validity of such a perturbation approach, the oscillation-centre approximation provides a good description of the exact solution. However, it has been shown that even for a modest field amplitude, the particle motion is unstable for frequencies in the vicinity of twice the cyclotron frequency and its sub-harmonics. In addition, the ponderomotive drift velocity calculated from numerical integration diverges not only if the applied frequency equals the cyclotron frequency, but also at the sub-harmonics of the cyclotron frequency. In the vicinity of these frequencies, the application of the oscillation-centre approximation is therefore clearly inappropriate.

The results of the present study reinforce the assertion of Sawley \& Václavik (1986) that the single-particle and fluid (collective) ponderomotive forces are fundamentally different. It has been shown that the oscillation-centre approximation is complete (within the limits of its application) in describing singleparticle behaviour under the influence of the perpendicular ponderomotive force. It is not possible to obtain the fluid result from single-particle theory (whether oscillation-centre approximation or exact solution). 
This work was partially supported by the Fonds National Suisse de la Recherche Scientifique.

\section{REFERENCES}

Fader, W. J., Jong, R. A., Stufflebeam, J. H. \& Sziklas, E. A. 1981 Phys. Rev. Lett. 46, 999.

Ferron, J. R., Hershkowitz, N., Breun, R. A., Golovato, S. N. \& Goulding, R. 1983 Phys. Rev. Lett. 51, 1955.

KLíma, R. 1968 Czech. J. Phys. B18, 1280.

McLachlan, N. W. 1964 Theory and Applications of Mathieu Functions. Dover.

Motz, H. \& Watson, C. J. H. 1967 Adv. Electron. Electron Phys. 23, 153.

Sawley, M. L. \& Václavík, J. 1986 Comments Plasma Phys. Contr. Fusion, 10, 19.

Statham, G. \& Ter HaAR, D. 1983 Plasma Phys. 25, 681.

Václavík, J., Sawley, M. L. \& Anderegg, F. 1986 Phys. Fluids, 29, 2034.

WATARI, T. et al. 1978 Phys. Fluids, 21, 2076.

YaSAKa, Y. \& Itatani, R. 1984 Nucl. Fusion, 24, 445. 